\title{
Discriminação entre rajadas de vento convectivas e não-convectivas
}

\author{
Discrimination between convective and non-convective wind gusts
}

\author{
Vanessa Ferreira e Ernani de Lima Nascimento
}

\begin{abstract}
Resumo
No presente estudo investigou-se detalhadamente uma série de 184 rajadas de vento originalmente descartadas por Ferreira e Nascimento (2015) por apresentarem um comportamento aparentemente não concordante com atividade convectiva local por persistirem por várias horas. O objetivo neste estudo foi confirmar ou não a natureza convectiva destas rajadas. Utilizando-se dados horários das estações meteorológicas de superfície do Instituto Nacional de Meteorologia (INMET), imagens do satélite Geostationary Operational Environmental Satellite (GOES 12 e 13), imagens de radares meteorológicos e dados de final analyse do modelo Global Forecast System (GFS-FNL) do National Centers for Environmental Prediction (NCEP) são analisados os 184 eventos de rajadas intensas, algums registrados por estações meteorológicas localizadas em altitudes elevadas e outros na região litorânea do sul brasileiro. Após uma análise criteriosa apenas 9 rajadas foram confirmadas como de origem convectiva. Para as estações de altitude o mecanismo forçante mais frequente associado às rajadas de vento intensas persistentes foi a presença de um escoamento de norte-noroeste tipo Jato de Baixos Níveis (JBN). Nas estações do litoral a maioria das rajadas intensas se originou de sistemas de baixa pressão em escala sinótica localizados próximos da costa do sul do Brasil, como ciclones extratropicais.
\end{abstract}

Palavras-chave: Rajada de vento, frente de rajada, tempestades, INMET, tempo severo.

\begin{abstract}
The present study ivestigated in detail a serie of 184 wind gusts events originally discarded by Ferreira e Nascimento (2015), which presented a behavior not consistent with local convective activity by persist for several hours. This study aims to confirm or not the convective nature of these wind gusts. Using hourly data from automated weather stations maintained by Brazil's National Weather Service (INMET), satellite imagery from the Geostationary Operational Environmental Satellite (GOES $12 \mathrm{e}$ 13), weather radars data and final analysis data from the National Centers for Environment Prediction (NCEP) Global Forecast System (GFS-FNL) the 184 intese wind gusts episodes was analysed, some recorded by weather stations located at high elevations and other in the coastal region of brazilian south. After a detailed analysis only 9 wind gusts events were confirmed as convective origin. For the weather stations located in high altitudes the most frequently forcing mechanism to explain intense wind gusts persisting for several hours was not convective activity, but the presence of a flow from north-northeast like a low-level jet (LLJ). In the coastal stations the most wind gusts originated from low pressure systems in synoptic scale located near the coast of southern Brazil, as extratropical cyclones.
\end{abstract}

Keywords: Wind gust, Gust front, Thunderstorm, INMET, Severe weather. 


\section{Introdução}

A Bacia do Prata, no setor subtropical da América do Sul, é uma das regiões do mundo mais favoráveis à ocorrência de tempestades convectivas severas (Nascimento, 2005; Brooks, 2006; Zipser et al., 2006; Cecil e Blankenship, 2012). Dentre os fenômenos que estas tempestades podem gerar estão as intensas rajadas de vento, que possuem um alto impacto para a sociedade devido ao seu potencial poder destrutivo. Entretanto, no Brasil, estudos sobre frentes de rajadas ainda são relativamente limitados (Lima e Menezes, 2004; Fogaccia e Filho, 2002).

O estudo de Ferreira e Nascimento (2015) (doravante FN15) explorou a detecção de frentes de rajadas originárias de tempestades severas pelas estações automáticas de superfície do Instituto Nacional de Meteorologia (INMET) na região sul do Brasil no período de 2005 a 2013. Naquele trabalho, um conjunto de critérios objetivos foi aplicado para selecionar somente rajadas de vento severas de origem convectiva. Porém, após a aplicação destes critérios, algumas estações meteorológicas apresentaram episódios suspeitos de não serem associados a tempestades convectivas locais devido ao registro consecutivo de rajadas intensas por várias horas, sugerindo a presença de uma forçante atmosférica de grande escala.

As estações em que estes eventos suspeitos foram observados estão localizadas em altitude ou no litoral. Existe a suspeita de que no primeiro caso muitas das rajadas de vento intensas e persistentes tenham resultado do efeito de montanha, com a estação meteorológica sendo exposta a ventos de altitude (de escala sinótica). No segundo caso a suspeita é de que muitas das rajadas intensas tenham se originado de sistemas de baixa pressão em escala sinótica localizados próximos da costa do sul do Brasil, como ciclones extratropicais por exemplo. Este trabalho dá continuidade a FN15 e investiga detalhadamente os eventos de rajadas de vento severas ocorridas no período de 2005 a 2014, que apresentaram comportamento aparentemente não concordante com atividade convectiva local. O objetivo é discriminar rajadas intensas geradas por tempestades convectivas locais daquelas que não são de origem convectiva. Esta abordagem é muito importante para o estudo da climatologia das tempestades severas locais no Brasil.

\section{Metodologia}

Para este estudo foram empregados dados meteorológicos horários registrados pelas estações meteorológicas automáticas de superfície do INMET durante o período de janeiro de 2005 a dezembro de 2014. Para efeito de contextualização, abaixo é descrito o método empregado por FN15 para a detecção de rajadas de vento intensas convectivas, seguido da metodologia específica usada no presente estudo.

Primeiramente, FN15 identificaram, para a região sul do Brasil e os setores dos estados de São Paulo e Mato Grosso do Sul ao sul de $22^{\circ} \mathrm{S}$, todos os eventos de rajadas de vento (rajada máxima da hora) com intensidade igual a ou maior que $25 \mathrm{~ms}^{-1}$. Este é o limiar tradicionalmente usado para definir rajadas de vento originadas de tempestades severas (Moller, 2001). Depois, imagens do canal infravermelho térmico do satélite Geostationary Operational Environmental Satellite (GOES 12 e 13) foram analisadas por FN15 a fim de verificar a presença de alguma tempestade convectiva sobre a estação meteorológica no intervalo de +/- 1 (uma) hora em torno do horário em que a rajada máxima de vento $\geq 25 \mathrm{~ms}^{-1}$ foi reportada. Para isto utilizou-se o valor de temperatura de brilho igual ou menor que $-55^{\circ} \mathrm{C}$ (aprox. $218 \mathrm{~K}$ ) como limiar para identificar a presença de convecção profunda sobre a estação (Matsudo e Salio, 2011; Salio et al., 2007; Machado et al., 1998).

Após a aplicação destes critérios, um escrutínio do comportamento horário das variáveis atmosféricas para os casos selecionados revelou, para quatro estações meteorológicas, um comportamento suspeito: o registro, por várias horas consecutivas, de rajadas máximas de vento $\geq 25 \mathrm{~ms}^{-1}$. As quatro localidades em que foi observado o comportamento suspeito foram: Morro da Igreja, localizada em uma região de topografia elevada em Santa Catarina, a $1780 \mathrm{~m}$ de altitude; São José dos Ausentes, localizada na região Serrana do Rio Grande do Sul, a uma altitude de $1229 \mathrm{~m}$; Mostardas, na região litorânea do Rio Grande do Sul; e a estação de Santa Marta, no litoral de Santa Catarina. Os eventos suspeitos de rajadas identificados para estas quatro estações foram (todos) descartados da análise realizada por FN15.

Nesta continuação, uma avaliação destes eventos é feita para verificar se dentre os casos descartados por FN15 existem rajadas genuinamente de origem convectiva. Além disto, foram incluídos eventos suspeitos registrados nos anos de 2010, 2011 e 2014, anos não estudados por FN15. Totalizou-se 184 eventos suspeitos. Em seguida, aplicou-se sobre estes eventos um novo critério de seleção de rajadas de vento convectivas que requer a ocorrência de precipitação acumulada na hora de registro da rajada, reduzindo-se este número para 119.

Em uma etapa seguinte, os eventos em que foram registradas rajadas máximas $\geq 25 \mathrm{~ms}^{-1}$ durante um intervalo de 3 (três) ou mais horas consecutivas foram descartados da amostra. É extremamente improvável que tempestades convectivas locais consigam gerar, sobre uma mesma estação meteorológica fixa, mais de 2 (dois) relatos horários consecutivos de rajadas máximas de vento acima de $\geq 25 \mathrm{~ms}^{-1}$. Estes eventos são tipicamente discretos e não sustentados por várias horas sobre um mesmo local (Fujita, 1986; Engerer et al., 2008). 
Após este descarte inicial, foi feita uma análise mais detalhada dos eventos remanescentes a fim de discriminar as rajadas de origem convectiva das não-convectivas. Para isto, o ambiente sinótico de cada evento foi estudado utilizando-se os dados da "análise final" (final analysis) do modelo Global Forecast System (GFS) do $\mathrm{Na}$ tional Centers for Environmental Prediction (NCEP) com espaçamento horizontal de $1^{\circ}$ de latitude/longitude, além de imagens de satélite e de radar meteorológico (quando disponíveis).

Para cada um dos eventos remanescentes, analisouse também as séries temporais horárias de rajada de vento, pressão atmosférica, temperatura do ar e precipitação para um intervalo de +/- 10 (dez) horas centrado em torno do horário em que a rajada mais intensa foi reportada (perfazendo então um intervalo de 21 horas). O objetivo com isto foi identificar padrões atmosféricos consistentes com piscinas de ar frio e mesoaltas, que acompanham as rajadas de vento de origem convectiva e que servem como um fator para diferenciar rajadas de origem convectiva e não-convectiva (FN15). Assim como em FN15, as séries temporais de temperatura e pressão foram estudadas utilizando-se os seus valores relativos, ou seja, as anomalias em relação aos seus respectivos valores médios no intervalo de 21 horas considerado. As séries de precipitação e rajada de vento foram analisadas simplesmente através do seu valor absoluto registrado em cada hora.

\section{Resultados e discussão}

Dos 119 episódios de rajadas suspeitas de não serem de origem convectiva, $72 \%$ foram registrados nas duas estações de montanha, o que aumenta a suspeita da influência da elevação das estações sobre estas medições, particularmente para a estação do Morro da Igreja detentora do maior número de eventos (66 eventos). De fato, após o descarte de todos os episódios com 3 (três) ou mais horas consecutivas com registros de rajadas máximas $\geq 25 \mathrm{~ms}^{-1}$, o número de eventos suspeitos (a serem avaliados com mais detalhe) foi reduzido de 119 para 35, e as duas estações de montanha foram as que mais tiveram registros de rajadas descartados, conforme mostra a Tabela 1 . Mais adiante serão apresentadas mais evidências de que a maioria das rajadas suspeitas nas estações de montanha realmente esteve associada a forçante sinótica.

Para ilustrar melhor o comportamento típico das variáveis atmosféricas durante uma rajada de vento severa de origem convectiva utiliza-se a Figura 1. Nela temos as séries temporais das rajadas máximas horárias, da precipitação horária e das anomalias de pressão atmosférica e de temperatura do ar em torno do horário de ocorrência de uma rajada de mais de $\geq 25 \mathrm{~ms}^{-1}$ advinda
Tabela 1: Número de eventos de rajadas suspeitos após a aplicação dos critérios descritos na metodologia deste trabalho.

\begin{tabular}{cccc}
\hline Critério & $\begin{array}{c}\text { Total de } \\
\text { eventos }\end{array}$ & $\begin{array}{c}\text { Estações } \\
\text { de altitude }\end{array}$ & $\begin{array}{c}\text { Estações } \\
\text { do litoral }\end{array}$ \\
\hline (FN15) & 184 & 112 & 72 \\
Precipitação & 119 & 85 & 34 \\
Rajada consec. & 35 & 24 & 11 \\
\hline
\end{tabular}

de uma tempestade convectiva registrada pela estação do INMET localizada em São Borja (RS) no dia 22 de julho de 2008 às 00 UTC.

Nota-se que as séries temporais das rajadas e da precipitação (Figura 1(a)) apresentam um máximo isolado, o que é um comportamento típico de eventos de caráter mais discreto, e condizente com uma tempestade convectiva local (Fujita, 1986). Em termos de pressão atmosférica (Figura 1(b)), há uma mudança na tendência do sinal, indo de negativo nas horas anteriores à rajada para positivo nas horas posteriores. Nota-se também um aumento mais abrupto da pressão dentro do intervalo de 1 (uma) hora em que a rajada foi reportada (hora zero). Em termos de anomalia de temperatura do ar (Figura 1(b)), há uma mudança de valores positivos antes da rajada para negativos após a rajada, com a queda mais acentuada da temperatura ocorrendo no horário em que a rajada foi reportada. A combinação do comportamento destas duas variáveis termodinâmicas com o da rajada de vento e da precipitação representa a manifestação da passagem da frente de rajada e dos padrões de mesoescala acompanhantes: a mesoalta e a piscina de ar frio (FN15).

Para muitas das rajadas de vento registradas nas estações de altitude um comportamento distinto do ilustrado na Figura 1 foi encontrado. De fato, nestas localidades o mecanismo forçante mais frequente para as rajadas de vento intensas persistindo por várias horas não foi atividade convectiva mas a presença de um escoamento de norte-noroeste tipo Jato de Baixos Níveis (JBN). Estas estações meteorológicas estão localizadas no (ou próximo ao) nível isobárico de $850 \mathrm{hPa}$. Logo, quando um escoamento tipo JBN impinge sobre a região da estação, a mesma amostrará por horas, intensas rajadas de vento que não são originárias de uma tempestade convectiva local (mesmo que eventualmente haja nebulosidade convectiva sobre a estação no horário de alguma(s) das rajadas, o que pode "confundir" o algoritmo de detecção de rajadas convectivas).

Para evidenciar isto de maneira mais clara, mostramos como exemplo o caso de 14 de maio de 2009 na estação do Morro da Igreja (Figura 2). O evento de rajada selecionado ocorreu às 00UTC, com intensidade de $29,9 \mathrm{~ms}^{-1}$. Nota-se pela série temporal de rajadas máxi- 


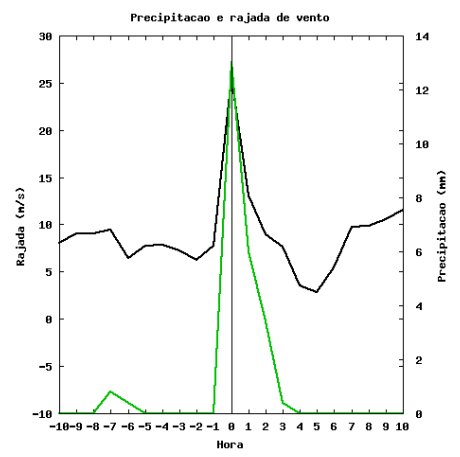

(a)

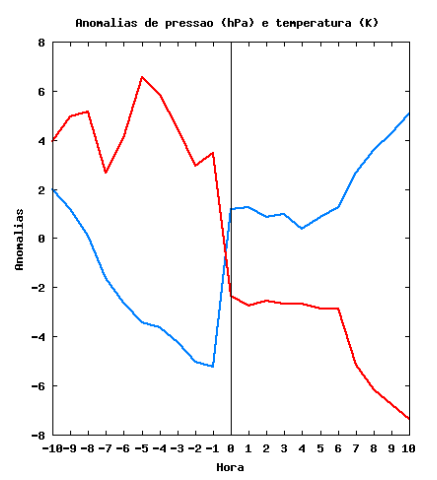

(b)

Figura 1: Séries temporais de: (a) rajada máxima horária $\left(\mathrm{ms}^{-1}\right)$ e precipitação horária acumulada $(\mathrm{mm})$, e (b) anomalias horárias de pressão atmosférica $(\mathrm{hPa}) \mathrm{e}$ temperatura do ar $(\mathrm{K})$, dentro do intervalo de +/- 10 horas centrado no horário de ocorrência dea uma rajada produzida por tempestade severa (indicado como hora 0 (zero) na abscissa das figuras). Evento de rajada registrado pela estação meteorológica do INMET em São Borja (RS) às 00 UTC do dia 22 de julho de 2008. As horas negativas [positivas] indicam o período antecedente [posterior] à rajada.

mas horárias (Figura 2(a)) a ocorrência de várias outras rajadas com velocidades de cerca de $20 \mathrm{~ms}^{-1}$ em torno do horário da rajada severa. A Figura 3 mostra a análise do modelo GFS em 850hPa válida às $00 \mathrm{Z}$ de 14/05/2009. A Serra Catarinense encontrava-se sob a ação de um escoamento de noroeste relativamente intenso, do tipo JBN.

Neste evento, a máxima precipitação horária acumulada não coincidiu com a hora da rajada máxima (Figura 2(a)). A série temporal das anomalias de pressão atmosférica (Figura 2(b)) mostra uma fraca queda da pressão na hora do registro da rajada, enquanto que a série de anomalias de temperatura do ar não apresenta uma tendência de queda na hora em que a rajada foi registrada. O comportamento combinado de todas estas variáveis não é consistente com a passagem de uma frente de rajada convectiva pela estação às 00UTC de 14 de maio de 2009 (contraste com a Figura 1 e com as figuras de FN15), não obstante o fato de haver um Sistema Convectivo de Mesoescala sobre a região naquele horário (não mostrado).

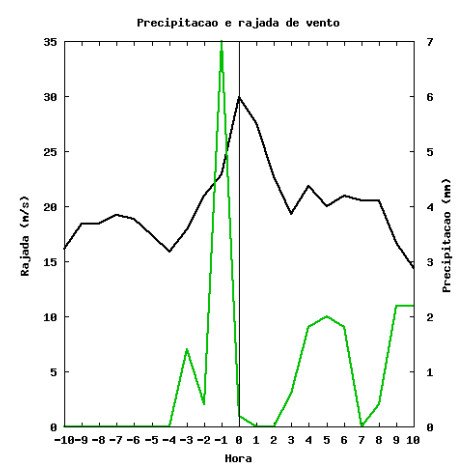

(a)



(b)

Figura 2: Igual a Figura 1, mas para a rajada intensa registrada pela estação meteorológica do INMET de Morro da Igreja (SC) às 00 UTC do dia 14 de maio de 2009.

Em contraste, o caso ilustrado na Figura 4 exemplifica um evento de rajada classificado como sendo de origem convectiva registrado pela estação de São José dos Ausentes, localizada a $1229 \mathrm{~m}$ de altitude, no dia 19 de outubro de 2014 as 08 UTC. A Figura 4(a) mostra que há um pico de velocidade da rajada relacionado ao evento considerado, e um segundo pico na oitava hora posterior a hora em que a rajada analisada foi registrada, este segundo pico de velocidade pode estar associado ao sistema de escala sinótica na qual o evento convectivo estava inserido. Esta hipótese é reforçada pelo comportamento da precipitação (Figura 4(a)), onde observa-se que ocorreu precipitação na hora da rajada, mas o pico secundário ocorre na hora posterior e o pico máximo também ocorre oito horas após a rajada analisada. As séries das anomalias de pressão atmosférica e temperatura do ar (Figura 4(b)) mostram que houve aumento da pressão e queda da temperatura na hora em que a rajada foi reportada. Uma característica que reforça o fato desta rajada ser de origem convectiva é a imagem 


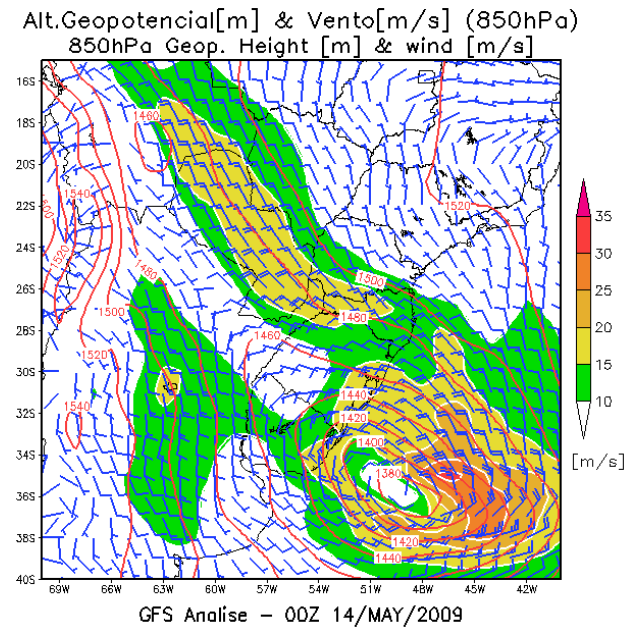

Figura 3: Altura geopotencial [isolinhas em mgp] e magnitude do vento $\left[\mathrm{ms}^{-1}\right]$ ( $\left(\right.$ sombreado acima de $5 \mathrm{~ms}^{-1}$ ) em $850 \mathrm{hPa}$ a para as $00 \mathrm{Z}$ de 14/05/2012. Análise do modelo GFS-NCEP.

de refletividade do radar localizado no Morro da Igreja (SC) (Figura 5), mostrando que havia um bow echo exatamente sobre São José dos Ausentes na hora em que a rajada intensa foi registrada pela estação meteorológica. Porém, para a maioria do total de 35 eventos analisado não haviam imagens de radar disponíveis.

Um evento de rajada de vento intensa não convectivo é mostrado na Figura 6, mas agora amostrado pela estação litorânea de Santa Marta (SC) as 06 UTC do dia 12 de maio de 2010. Novamente, observa-se que a estação registrou rajadas intensas por várias horas consecutivas, não apresentando um pico isolado de velocidade da rajada (Figura 6(a)). O comportamento da precipitação (Figura 6(a)) também não apresenta um pico isolado e pode-se notar que ocorreu precipitação em praticamente todo o período de 21 horas analisado. A Figura 6(b) mostra que a pressão atmosférica se manteve em queda nas horas anteriores e posteriores a hora da rajada. Já as anomalias de temperatura do ar (Figura 6(a)) mostram que ocorreu aumento da temperatura na hora em que a rajada foi reportada. O comportamento destas variáveis não está de acordo com aquele esperado para um evento de rajada de vento de origem convectiva. Estas rajadas registrada por esta estação podem ser explicadas devido a existência de um ciclone extratropical localizado próximo a costa de Santa Catarina (Figura 7).

Na Figura 8 observa-se um episódio de rajada de vento intensa registrado pela estação de Mostardas, localizada no litoral do Rio Grande do Sul, as 21 UTC do dia 29 de fevereiro de 2012. Este evento foi classificado como sendo de origem convectiva. Na Figura 8(a) vê-se que ocorreu um pico isolado de velocidade da rajada, e que esta foi acompanhada por precipitação. A análise das séries de pressão atmosférica e temperatura (Figura

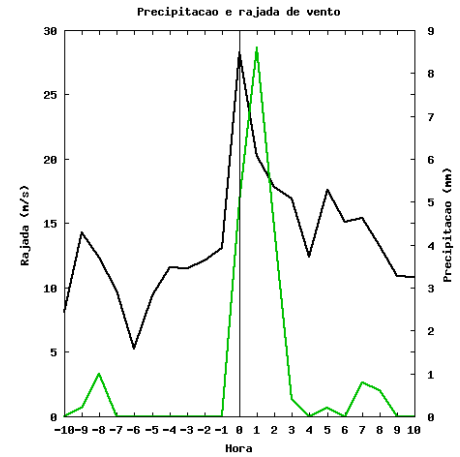

(a)

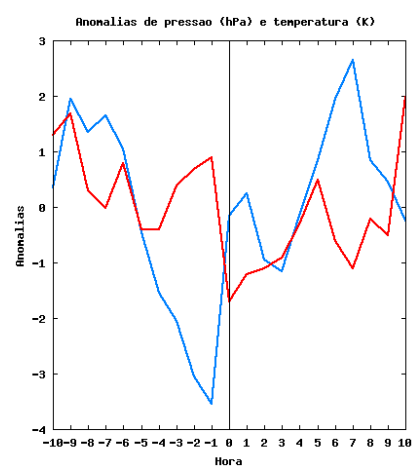

(b)

Figura 4: Igual a Figura 1, mas para a rajada intensa registrada pela estação meteorológica do INMET de São José dos no dia 19 de outubro de 2014 as 08 UTC.

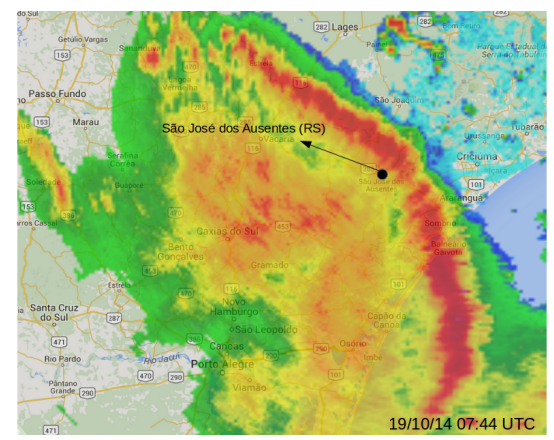

Figura 5: Imagem de refletividade do radar localizado no Morro da Igreja (SC) da REDEMET as 07:44 UTC de 19/10/2014. Fonte: REDEMET.

8(b) mostra que ocorreu aumento da pressão e queda da temperatura na hora em que a rajada foi reportada. A imagem do radar localizado na cidade de Canguçu (RS) (Figura 9) mostra que havia uma célula mais intensa que passou sobre Mostardas em um horário próximo ao qual a rajada foi reportada, reforçando a origem convectiva da rajada.

Após uma investigação detalhada dos 35 episódios suspeitos de rajadas de vento, apenas 9 (nove) foram classificados como eventos de origem convectiva. 


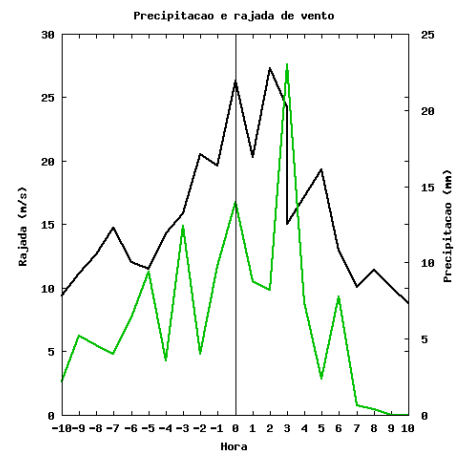

(a)

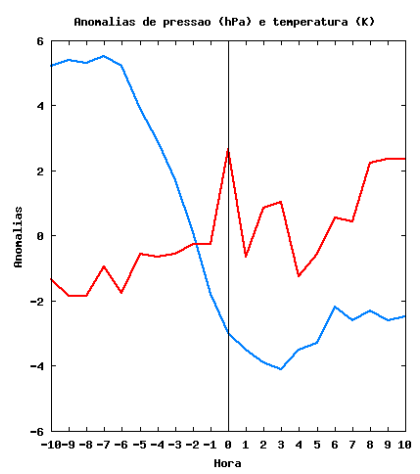

(b)

Figura 6: Igual a Figura 1, mas para a rajada intensa registrada pela estação meteorológica do INMET de Santa Marta as 06 UTC do dia 12 de maio de 2010.

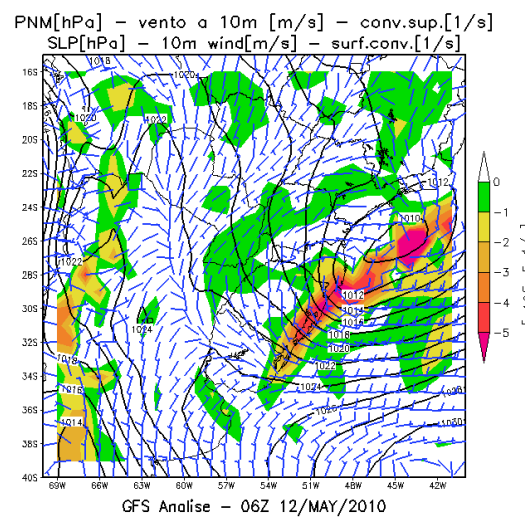

Figura 7: Pressão ao nível médio do mar [hPa] em

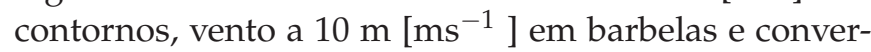
gência em superfície [ $\left[\mathrm{s}^{-1}\right]$ em sombreado para as $06 \mathrm{Z}$ de 12/05/2010. Análise do modelo GFS-NCEP.

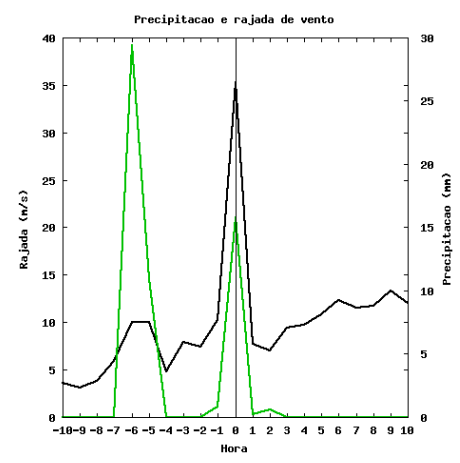

(a)

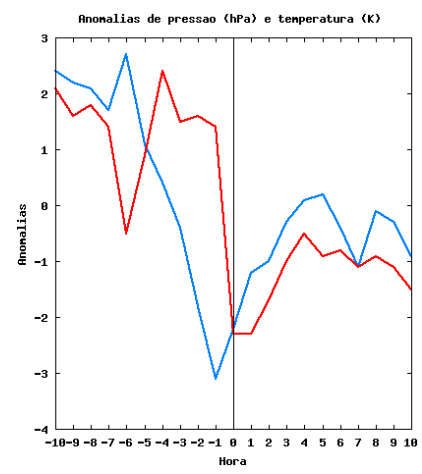

(b)

Figura 8: Igual a Figura 1, mas para a rajada intensa registrada pela estação meteorológica do INMET de Mostardas (RS), as 21 UTC do dia 29 de fevereiro de 2012.

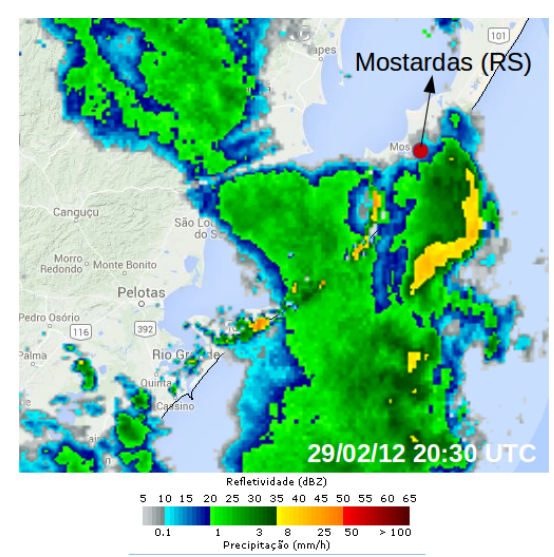

Figura 9: Imagem de refletividade do radar localizado Canguçu (RS) as 20:30 UTC de 29/02/2012 obtida do site da DSA/CPTEC. 


\section{Conclusões}

Pode-se concluir que a maioria das rajadas de vento intensas registradas pelas quatro estações meteorológicas analisadas neste estudo não são rajadas geradas por tempestades convectivas locais. Para as duas estações localizadas em regiões de altitude elevada, Morro da Igreja (SC) e São José dos Ausentes (RS), o principal fator de escala sinótica que está relacionado ao registro de rajadas intensas é um escoamento tipo JBN. Para as estações localizadas em regiões litorâneas, Santa Marta (SC) e Mostardas (RS), o registro de rajadas intensas de vento parece estar relacionado à ocorrência de um ciclone extratropical próximo à costa no Sul do Brasil.

Não foi possível estabelecer um único critério que diferenciasse rajadas de vento intensas de origem convectiva e de origem não convectiva. No entanto, mostrou-se que é possível diminuir consideravelmente a quantidade de eventos suspeitos de não serem de origem convectiva aplicando-se o conjunto critérios descrito na metodologia deste trabalho. Mostrou-se também que através da análise conjunta dos dados registrados pela estação meteorológica, investigação do ambiente sinótico e de dados de satélite e radar pode-se determinar se a rajada de vento foi ou não gerada por uma tempestade convectiva local.

\section{Referências}

Brooks, H. (2006). A global view of severe thunderstorms: Estimating the current distribution and possible future changes, preprints. Em: AMS Severe Local Storms Special Symposium, J4, p 2.

Cecil, D. J., Blankenship, C. B. (2012). Toward a global climatology of severe hailstorms as estimated by satellite passive microwave imagers. Journal of Climate, 25(2), 687-703.

Engerer, N. A., Stensrud, D. J., Coniglio, M. C. (2008). Surface characteristics of observed cold pools. Monthly Weather Review, 136(12), 4839-4849.

Ferreira, V., Nascimento, E. d. L. (2015). Severe wind gusts generated by thunderstorms as sampled by inmet $\mathrm{s}$ surface automated weather stations in southern brazil. Em: 14th International Conference on Wind Engineering.

Fogaccia, C. V. C., Filho, P. A. (2002). Turbulência e cisalhamento do vento na área do aeroporto internacional de são paulo/guarulhos. Em: XII Congresso Brasileiro de Meteorologia, Foz do Iguaçu, PR. Anais... Soc. Bras. Meteorologia.
Fujita, T. T. (1986). Mesoscale classifications: their history and their application to forecasting. Mesoscale Meteorology and Forecasting, P S Ray (Ed), American Meteorological Society, pp. 18-35.

Lima, D., Menezes, W. F. (2004). Queda de estruturas de linha de transmissão de energia elétrica em cachoeira paulista (sp) ea ocorrência de tempestades severas-um estudo de caso. Em: CONGRESSO BRASILEIRO DE METEOROLOGIA, XIII.

Machado, L., Rossow, W., Guedes, R., Walker, A. (1998). Life cycle variations of mesoscale convective systems over the americas. Monthly Weather Review, 126(6), 1630-1654.

Matsudo, C., Salio, P. (2011). Severe weather reports and proximity to deep convection over northern argentina. Atmospheric Research, 100(4), 523-537.

Moller, A. R. (2001). Severe local storms forecasting. Meteorological Monographs, 28(50), 433-480.

Nascimento, E. d. L. (2005). Previsão de tempestades severas utilizando-se parâmetros convectivos e modelos de mesoescala: uma estratégia operacional adotável no brasil. Revista Brasileira de Meteorologia, 20(1), 121140.

Salio, P., Nicolini, M., Zipser, E. J. (2007). Mesoscale convective systems over southeastern south america and their relationship with the south american lowlevel jet. Monthly Weather Review, 135(4), 1290-1309.

Zipser, E. J., Liu, C., Cecil, D. J., Nesbitt, S. W., Yorty, D. P. (2006). Where are the most intense thunderstorms on earth? Bulletin of the American Meteorological Society, 87(8), 1057-1071. 